\title{
A METHOD TO REDUCE HANDOFF DELAY FOR IMPROVING TCP/IP PERFORMANCE IN NEXT GENERATION WIRELESS NETWORKS
}

\author{
A.Ezil Sam Leni ${ }^{1}$, S.K Srivatsa ${ }^{2}$ \\ ${ }^{1}$ Research Scholar, Department of Computer Science and Engineering, \\ Sathyabama University, Rajiv Gandhi Road, Chennai, India \\ ${ }^{2}$ Senior Professor, St.Joseph's College of Engineering, Rajiv Gandhi Road, Chennai, India \\ Email: 'lenisatish@gmail.com
}

\begin{abstract}
Next Generation Wireless Networks demand for 'anywhere and anytime' access to high-speed data for real and non-real time multimedia services. Providing seamless handoff between different wireless access networks plays a vital role. In this paper, we propose a new handoff mechanism based on the information from the physical layer, data link layer, network layer and application layer. The collected information is used in execution of three stages in the handoff process of NGWS. The neighboring BSs of the moving MN are determined. The TOPSIS method is used to select the best available network based on minimum handoff signaling delay and QoS parameters of four types of wireless applications. The handoff initiation time is estimated dynamically and handoff is executed. Simulation results shows that the proposed mechanism gives reduced handoff delay when compared to the mechanism with layer (2+3) information.
\end{abstract}

Key words: Next Generation Wireless Networks, Handoff, TOPSIS, QoS parameters, Wireless Application Types.

\section{INTRODUCTION}

TCP/IP is the most prominent protocol in the internet. Today $90 \%$ of the Internet traffic is carried out by the TCP/IP. TCP/IP was initially developed for fixed-wired networks. Wired networks are with relatively reliable links and low bit error rate. With the rapid growth of wireless data services, TCP/IP has been widely deployed in wireless networks. In wireless networks, wireless channel is generally characterized by its high bit error rate. When TCP/IP is used in wireless networks, it gives poor performance. TCP/IP experiences transmission-error related packet loss called corruption loss and congestion related packet loss called congestion loss in wireless channels. In wireless channel, due to high BER, corruption loss occurs more frequently when compared to congestion loss. The main reason for poor performance of TCP in wireless networks is that TCP congestion control mechanism cannot differentiate between corruption loss and congestion loss in wireless environment.

Improving TCP/IP performance in wireless networks has been an active research [10][12]. Now the popularity of wireless networking has increased dramatically over the past few years. There is also a need for integrating high delay, high channel error wireless networks. In Next Generation Wireless Systems (NGWS), mobile users are demanding 'anywhere and anytime' access to high-speed data for real and non-real time multimedia services. These service requirements are in terms of latency, bandwidth and error rate. Moreover NGWS are designed with specific service needs and vary widely in terms of bandwidth, latency, area of coverage, cost and QoS provisioning. The NGWS architecture is mainly to integrate heterogeneous wireless networks. Heterogeneous wireless networks are optimized for some specific service demands and coverage area. In this regard, the mobile user is always connected to the best available network. It is necessary to consider about the performance of wireless network in NGWS.. Improving the performance of the TCP/IP in such heterogeneous wireless networks to provide seamless mobility poses a significant challenge in research community

The paper is organized as follows: Section-2 describes the survey of related work. In Section-3, the architecture, flow and implementation of the proposed mechanism is explained. The performance of the proposed mechanism is analyzed and simulation results are shown in Section-4.We conclude the paper in Section-5.

\section{SURVEY OF RELATED WORK}

In the literature, there exist several solutions regarding Handoff [3] [4] [12] [8]. In [8], a new mechanism known as Receiver-Based Vertical Handover is proposed. This is based on the Bandwidth Delay Product (BDP) measurement and congestion control using the receiver's advertised window. Make-Before-Break (MBB) handoff mechanism is used in the receiver to enable seam less handoff. The handoff scenario is considered between $3 G$ wireless networks, GPRS and WLAN. 4G wireless system, are expected to provide high data rate, QoS guaranteed, seamless multimedia connections [12]. The technical challenges in $4 \mathrm{G}$ wireless networks are: security, routing, mobility management and radio resource management. These aspects are described in [13].A downlink queuing model based on two-layer is proposed. The queuing model based on Radio layer and IP layer is designed to provide high system throughput and system performance. In [4], a 
new handoff management protocol for next generation wireless networks, to support seamless handoff management is proposed. The proposed handoff management is based on the data link layer and network layer information to initiate when to begin with handoff. Performance analysis of the existing handoff technique is studied in [2]. The performance of these protocols varies with different applications. Performance of Mobile IP, TCPMigrate and SIP are analyzed in this paper. Using network layer based mobility management protocol Mobile IP [5] and Hierarchical Mobile IP [7] are discussed. In the analysis it is stated that Mobile IP latency results in significant packet losses in handoff. In IP based wireless networks IDMP [14] (Intra-domain Based Mobility Protocol), (CIP) Cellular IP [16] and HAWAll [15] are used as the mobility management protocol. Cellular IP provides local mobility and handoff support for frequently moving hosts. It also supports fast handoff and paging in CIP access networks. IDMP is a two-level hierarchical approach to provide mobility support for MNs in IP-based mobile networks. In [1] an algorithm is proposed to estimate the velocity of the mobile device using power spectral density. Researchers proposed algorithm for initiating handoff process using the momentum and is discussed[9].

\section{PROPOSED MECHANISM}

\subsection{NetworkArchitecture}

Rapid progress in the research and development of wireless networking and communication technologies has created several wireless communication systems such as GSM (Global System for Communication), GPRS(General Packet Radio Services), UMTS(Universal Mobile Telecommunication Services) and Wireless LAN (WLAN), Bluetooth for personal area networking and Satellite networks for Global networking. These networks complementary to each other and hence their integration can realize unified Next-Generation Wireless Systems (NGWS) i.e. 4G Networks. In the integrated NGWS, users are always connected to the best available networks and switch between different networks based on their service needs [14]. A challenging issue in NGWS is to develop a handoff algorithm for NGWS. The architecture of the $4 G$ wireless networks is given in fig.1.

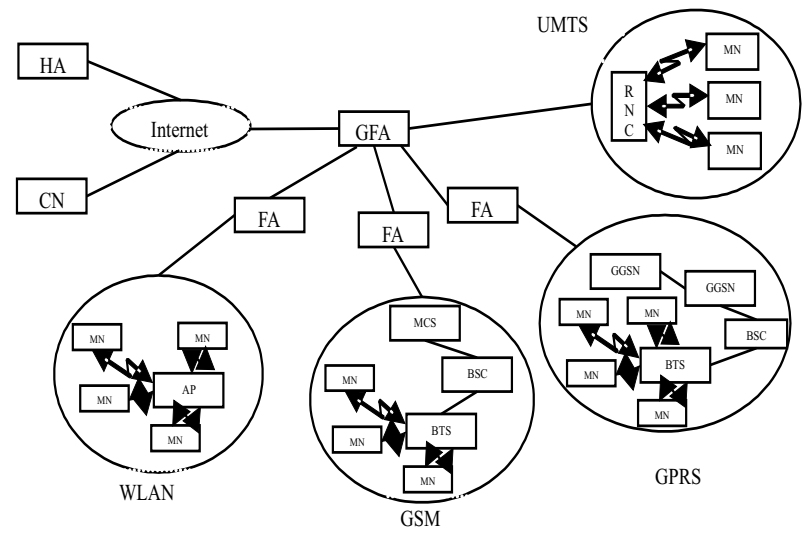

Fig. 1.Fourth Generation Network Architecture

\subsection{System Model}

The system model for the proposed technique is given in fig.2. The layer information from the physical layer, data link layer, network layer and application layer are given to the monitoring unit. The monitoring unit periodically gives the information to the optimization unit. Optimization unit uses TOPSIS method to select the best network with minimum handoff signaling delay and specific QoS parameters to the applications.

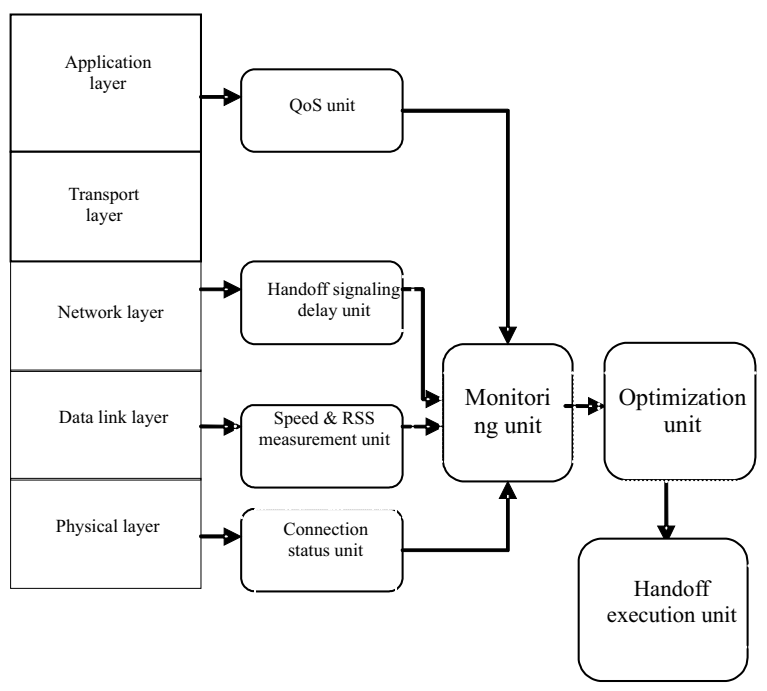

Fig. 2.The Proposed System Architecture

The functions of each unit are as follows:

\section{Physical Layer}

Physical Layer monitors the status of the physical connection. Two state Markov chain model is used to identify the channel as good state or bad state. According to the Markov chain model, if the BER is very high, the 
channel is said as Bad Channel, and if the BER is low, the channel is said as Good Channel. This information is stored in the connection status unit and periodically monitored information about the channel is given to the Monitoring unit.

\section{Data Link Layer}

The velocity of the mobile user is used in this layer to predict, when handoff is to be initiated. The VEPSD[1] algorithm is used to estimate the speed of the mobile user. This layer is also used to measure the RSS of the mobile user based on the speed of the mobile user. If the measured RSS of the mobile node drops below the threshold value $t_{\text {rss, }}$ the handoff procedure is executed. These information is available in the Speed and RSS measurement unit and periodically given to the Monitoring unit.

\section{Network Layer}

This Layer estimates the handoff signaling delay of the mobile user with the neighboring BSs. There are several algorithms in the literature for finding the neighboring BSs. Candidate Access Route Discovery (CARD) is used to learn about the neighboring BSs. The delay associated with the intersystem handoff is calculated. An important point to be considered handoff signaling delay estimation is, the handoff signaling delay is not a constant value. It increases if the user is far from the Home Network $(\mathrm{HN})$ or the network experiences high delay. Handoff signaling delay also varies with network dynamics like Congestion level, wireless link condition, location of user from $\mathrm{HN}$ etc.

Application layer

According to 3GPP, there are four different applications in wireless networks namely, Conversational, Streaming, Interactive and Background traffic. In application layer, the information about the type of application and the related QoS parameters are obtained and given to the monitoring unit.

\section{Monitoring Unit}

Monitoring unit collects information from the Connection status unit, RSS and Speed Estimation unit, handoff signaling delay estimation unit and application unit. The collected information is given to the Optimization unit.

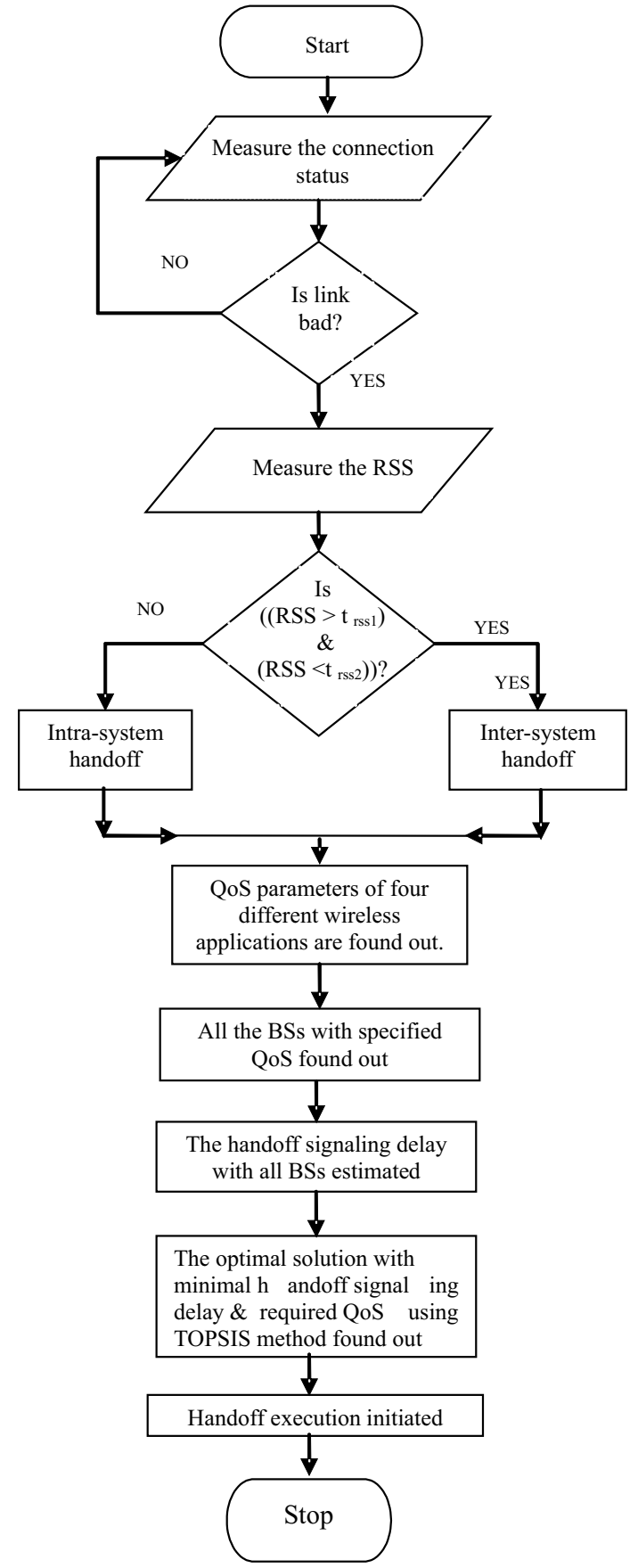

Fig. 3. Processing flow of the proposed system Optimization Unit:-

The main function of the optimization unit is to select the best available network, that gives minimum handoff delay and to satisfy the QoS parameters specific to the application. TOPSIS method is used to find the optimal solution i.e. the best network based on the given criteria. 
Handoff Unit

From the optimization unit, it gives the details about the best available network with minimum handoff signaling delay and specific QoS parameters towards the direction of the mobile user. Then the handoff unit executes handoff process.

\subsubsection{Stages in Handoff Process in NGWS}

In the NGWS the handoff process is divided into three stages namely

\section{System Discovery}

Handoff Decision

Handoff Execution

\subsubsection{System Discovery}

In the system discovery stage, the neighboring network of the current mobile device is identified. In the proposed technique CARD (Candidate Access Router Discovery) protocol is used to identify the neighboring Base Stations.

\subsubsection{HandoffDecision}

In the Handoff Decision stage, it determines which network can be used to connect to, based on different parameters. In the proposed technique, Handoff Signaling Delay, Jitter, Delay, Packet Loss Rate and Maximum bit rate are taken as the criteria for selecting the best network. TOPSIS method is used to select the best available network based on the above parameters.

\subsubsection{HandoffExecution}

In the Handoff Execution stage, the time to initiate the handoff process is calculated and then the handoff process is executed by establishing connection to the selected network. In the proposed technique we used Mobile IP protocol.

The various steps in the proposed handoff mechanism are shown in fig.3.

\subsubsection{Best Network Selection-TOPSIS Method}

TOPSIS (Technique for Order Preference by Similarity to the Ideal Solution), selects a network that satisfies the given criteria after performing six sequential steps listed below. The network with maximum value from the rank order is the one that is closest to the ideal solution and far away from the negative ideal solution. In the proposed technique, as the alternative WLAN, GSM, GPRS and UMTS are taken. The criteria for selecting the best network are Handoff Signaling Delay, Jitter, Delay, Packet Loss
Rate and Maximum bit rate. In this, handoff signaling delay, Jitter, Delay and Packet loss rate are to be minimal and bit rate is to be maximum. The TOPSIS algorithm is implemented in $\mathrm{C}$ and the results are analyzed. In 3GPP wireless applications are divided into four different traffic classes namely, conversational, streaming, interactive and Background. For each of this application QoS parameters are defined in 3GPP and are used in the network selection. The steps in TOPSIS method are given below.

Construct the normalized decision matrix

Construct the weighted normalized decision matrix

Determine the ideal and Negative -ideal solution

Calculate the separation measure

Calculate the relative closeness to the ideal solution

Rank the preference order

The best alternative is obtained from the preference order and is the one that has the shortest distance to the ideal solution and have the longest distance to the negative ideal solution. The various measures and values obtained through simulation are shown in Table.1, Table.2 and in Table.3.

Table 1. Simulation Parameters

\begin{tabular}{|c|c|c|c|c|c|}
\hline $\begin{array}{c}\text { Alternatives/ } \\
\text { Criteria }\end{array}$ & $\begin{array}{c}\text { HO Delay } \\
(\mathrm{ms})\end{array}$ & $\begin{array}{c}\text { Jitter } \\
(\mathrm{ms})\end{array}$ & $\begin{array}{c}\text { Delay } \\
(\mathrm{ms})\end{array}$ & $\begin{array}{c}\text { Packet } \\
\text { Loss Rate } \\
(\mathrm{ms})\end{array}$ & $\begin{array}{c}\text { Max.Bit } \\
\text { Rate } \\
(\mathrm{Mbps})\end{array}$ \\
\hline WLAN & 171 & 3 & 160 & 0.01 & 5.4 \\
\hline GPRS & 175 & 5 & 164 & 0.001 & 5.4 \\
\hline GSM & 163 & 6 & 167 & 0.01 & 5.4 \\
\hline UMTS & 156 & 4 & 160 & 0.001 & 5.4 \\
\hline
\end{tabular}

Table 2. Ideal and Negative Ideal Solution

\begin{tabular}{|l|c|c|c|c|c|}
\hline \begin{tabular}{|l|l|l|} 
Alternative \\
/Criteria
\end{tabular} & $\begin{array}{c}\text { HO Delay } \\
(\mathrm{ms})\end{array}$ & $\begin{array}{c}\text { Jitter } \\
(\mathrm{ms})\end{array}$ & $\begin{array}{c}\text { Delay } \\
(\mathrm{ms})\end{array}$ & $\begin{array}{c}\text { Packet } \\
\text { Loss Rate } \\
(\mathrm{ms})\end{array}$ & $\begin{array}{c}\text { Max.Bit } \\
\text { Rate } \\
(\mathrm{Mbps})\end{array}$ \\
\hline WLAN & 0.201692 & 0.048525 & 0.073953 & 0.105540 & 0.07500 \\
\hline GPRS & 0.206410 & 0.080875 & 0.075801 & 0.010554 & 0.07500 \\
\hline GSM & 0.200512 & 0.097049 & 0.076264 & 0.105540 & 0.07500 \\
\hline UMTS & 0.191076 & 0.064700 & 0.064700 & 0.010554 & 0.07500 \\
\hline $\begin{array}{l}\text { deal } \\
\text { Solution }\end{array}$ & 0.191076 & 0.048525 & 0.073953 & 0.010554 & 0.07500 \\
\hline $\begin{array}{l}\text { Negative } \\
\text { deal } \\
\text { Solution }\end{array}$ & 0.206410 & 0.097049 & 0.076264 & 0.105540 & 0.07500 \\
\hline
\end{tabular}


Table 3. Relative Closeness to the Ideal Solution

\begin{tabular}{|c|c|c|c|}
\hline $\begin{array}{c}\text { Alternatives/ } \\
\text { Separation } \\
\text { Measure }\end{array}$ & Ideal Solution & $\begin{array}{c}\text { Negative } \\
\text { Ideal } \\
\text { Solution }\end{array}$ & Relative Closeness \\
\hline WLAN & 0.095577 & 0.048808 & 0.338042 \\
\hline GPRS & 0.035847 & 0.096354 & 0.728842 \\
\hline GSM & 0.107104 & 0.005897 & 0.052189 \\
\hline UMTS & 0.016175 & 0.101534 & 0.862586 \\
\hline
\end{tabular}

Once the best network which satisfies the given criteria is selected, the handoff initiation time is calculated and the handoff process is executed.

\section{SIMULATION}

\subsection{Simulation Environment}

The proposed system is simulated in ns-2 simulator and the results are analyzed. The network scenario taken for simulation is shown in fig. 4 .

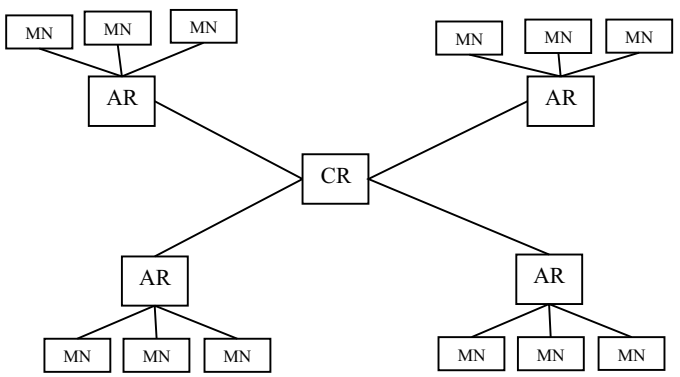

Fig. 4. Simulation Structure

\subsection{Simulation Results}

The performance of the four different types of application is analyzed and the results are compared with the layer $(2+3)$ method. Conversational traffic, Streaming traffic, Interactive traffic and background traffic are considered during simulation.

4.2.1 Performance of the proposed mechanism with handoff signaling delay

The relationship between the number of handoffs and the handoff signaling delay is shown in Fig 5. Handoff signaling delay depends on the traffic load in the backbone network, wireless link quality, distance between user and its Home Network $(\mathrm{HN})$ at the handoff instance, user speed on the performance of the handoff protocols. When the number of handoff increases, the handoff signaling delay also increases. The performance of the proposed mechanism is compared with the layer $(2+3)$ based handoff, and the simulation result shows that when the number of handoffs increases the handoff signaling delay also increases. The increase in value is lower than that of the layer $(2+3)$ mechanism.

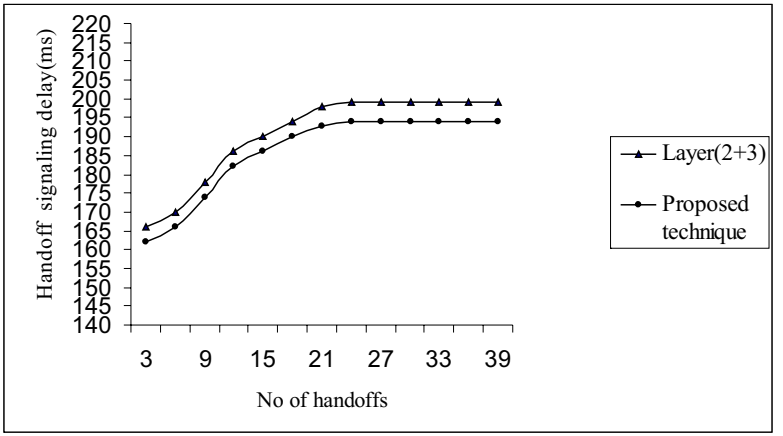

Fig. 5. Relationship between number of handoff and handoff signaling delay

4.2.2 Performance of the proposed mechanism with handoff failure probability

Relationship between the number of handoffs and the handoff failure probability is shown in Fig. 6 . Once the speed of the mobile device is estimated, based on the channel quality, measured RSS and QoS parameters from the application layer the best network is selected, and then the handoff is executed in the network layer. Figure 6 shows that due to this constraint, there is a recognizable degradation in the handoff failure probability. When the number of handoffs has less value, the handoff failure probability started from 0.02 . In the proposed technique, when the number of handoff increases the handoff failure probability gradually increased. When the number of handoffs performed is more than 15 , there is an exponential increase in handoff failure probability and after certain time, the handoff failure probability remains the same. When the number of handoffs is increased, the hand off signaling delay and handoff delay increases due to the current traffic load. Thus the simulation result shows that the proposed technique gives better performance compared with the handoff based on Layer $(2+3)$ information.

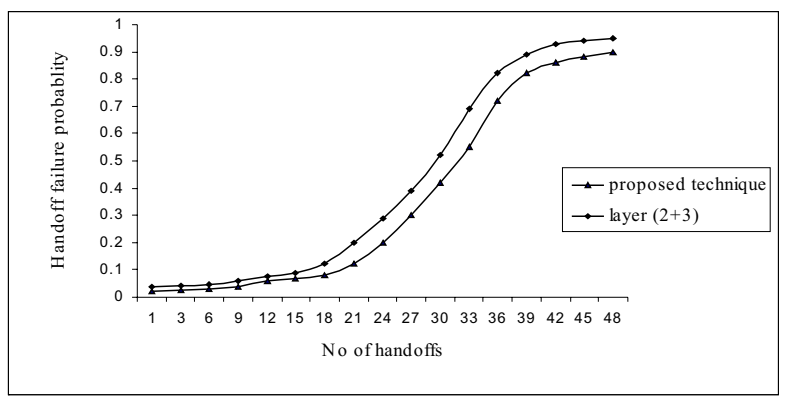

Fig.6. Relationship between number of handoff and handoff failure probability

\subsubsection{Performance of Network Selection}

During the simulation run in TOPSIS method the best network is selected based on the QoS parameters and the minimum handoff signaling delay. The percentage of time a particular network is selected is shown in fig. 7 . 


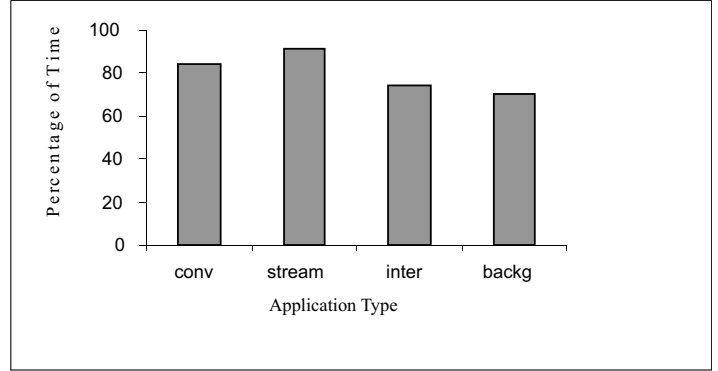

Fig. 7. Percentage of time the same network selection

\section{CONCLUSION}

In this paper, the existing work done in the mobility management in wireless networks is studied. We propose a new handoff mechanism based on the information from the Physical Layer, Data Link Layer, Network Layer and Application Layer. In the physical layer connections status is measured. The speed of the mobile device and RSS is estimated in the Data Link Layer. Network Layer first determines the neighboring BSs using CARD protocol and then estimates the handoff signaling delay. From the application layer the QoS parameters of the four different applications are obtained. This information is monitored periodically in the monitoring unit. The best network based on the given requirements is selected using TOPSIS method. The handoff is executed with the selected network. The performance of the proposed mechanism is compared with the layer $(2+3)$ mechanism and the simulation results are obtained.

\section{REFERENCES}

[1] Shantidev Mohanty, "VEPSD:A Novel Velocity Estimation Algorithm for Next-Generation Wireless Systems", IEEE Transactions on Wireless Communications, Vol.4, No.6, November 2006.

[2] Shantidev Mohanty and lan F. Akyildiz, "Performance Analysis of Handoff Techniques Based on Mobile IP, TCP-Migrate, and SIP", IEEE Transactions on Mobile Computing, Vol.6, No.7, July 2007.

[3] lan F. Akyildiz Jiang Xie, and Shantidev Mohanty, "A Survey of Mobility Management in Next-Generation All-IP-Based Wireless Systems", IEEE Wireless Communications, August 2004.

[4] Shantidev Mohanty and lan F.Akyildiz, "ACross-Layer (Layer $2+3$ ) Handoff Management Protocol for NextGeneration Wireless Systems", IEEE Transactions on MobileComputing, Col.5, No.10, October 2006.

[5] Perkins C etal., "IP Mobility support for IPv4", Internet Engineerig Task force RFC 3344,Aug.2002

[6] Akyyildiz I.F, Mohanty S, and Xie J, "A Ubiquitous Mobile Communication Architecture for Next Generation Heterogeneous Wireless systems, "IEEE Radio comm., Magazine, vol.43, . .6, pp.s29s36, June 2005.
[7] H.Soliman, C.Castelluccia and K.El-Malki, "Hierarchical Mobile IPv6 Mobility Management ," IETF Internet Draft, http://www3.ietf.org/ proceedings/02mar//-D/draft-ietf-mobileip-hmipv605.txt, July 2001

[8] Yuci Gou, David A, J.Pearce, "A Receiver -based Vertical Handover Mechanism for TCP Congestion Control", IEEE Transactions on Wireless Communications, Vol.5, No.10, October 2006.

[9] Tein-Yaw chung, chih-Hung Hsu, Yung-Mu Chen, and K.Robert Lai, "Momentum-based motion Methodology for Handoff in Wireless Networks", 22 nd International Conference on Advanced Information Networking and Applications.

[10] V.Mariappan, R.Padhmapriya, and P.Narayanasamy, "QoS Enabled Dynamic Handoff in wireless Networks",

[11] Nilanjan Banerjee, Sajal K.Das and Arup Acharya, "SIP-based Mobility Architecture for Next Generation Wireless Networks", Proceedings of the $3^{\text {rd }}$ IEEE international Conference on Pervasive Computing and Communications (PerCom 2005).

[12] Stefan alfredsson, Anna Brunstrom and Mikael Sternad, "Cross-Layer analysis of TCP performance in a 4G system",

[13] H.N.Nguyen and I.Sasase, "Downlink Queuing Model and Packet scheduling for providing lossless handoff and QoS in 4G Wireless Networks", IEEE Transactions Algorithm for Fourth Generation Mobile Networks," IEEE Transactions on Mobile Computing.

[14] A Misraet.al, "IDMP-BasedFastHandoffs and Paging in IP-Based 4G Mobile Networks," IEEE Communication magazine March.2002, pp.138-145.

[15] R.Ramjee, "HAWAll: A Domain Based Approach Supporting Mobility in Wide-Area Networks, IEEE/ACM Transactions on Networking., volume 10, no.3, June 2002, pp.396-410.

[16] A.T Campbell et.al, "Design, Implementation, and evaluation of Cellular IP," IEEE Personal Communication., August 2000, pp.42-49.

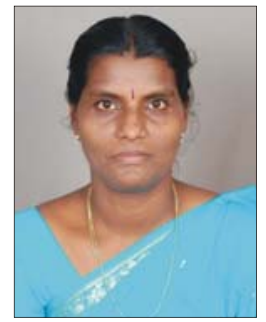

\section{A.Ezil Sam Leni}

She is currently working as an Assistant Professor in the Department of Computer Science and Engineering at Sathyabama University, Chennai. She has 12 years of teaching experience. She is a life time member of ISTE. Her current areas of interests include performance enhancement of TCP/IP protocol in wireless networks, mobility management and QoS in next generation wireless networks. 